\title{
Evaluation of laparoscopic-assisted transanal total mesorectal excision(ta-TME) for locally advanced, distal rectal cancer in men with difficult pelvic conditions after neoadjuvant chemoradiation therapy
}

\section{Yingjie Li}

Beijing Cancer Hospital

Guoli He

Nanxishan Hospital of Guangxi Zhuang Autonomous Region

Lin Wang

Beijing Cancer Hospital

Qiushi Dong

Beijing Cancer Hospital

Xinzhi Liu

Beijing Cancer Hospital

Jiahua Leng

Beijing Cancer Hospital

Xiao Zhang

Beijing Cancer Hospital

Tingting Sun

Beijing Cancer Hospital

Yue Zhang

Beijing Cancer Hospital

\section{Yunfeng Yao}

Beijing Cancer Hospital

Aiwen Wu ( $\nabla$ drwuaw@sina.com )

Beijing Cancer Hospital https://orcid.org/0000-0003-1877-7005

Research article

Keywords: Ta-TME, difficulty pelvis, rectal cancer surgery, sphincter-preserving proctectomy

Posted Date: August 25th, 2020

DOI: https://doi.org/10.21203/rs.3.rs-50257/v1 
License: (c) (i) This work is licensed under a Creative Commons Attribution 4.0 International License. Read Full License 


\section{Abstract}

\section{Background}

To evaluate the use of laparoscopic-assisted transanal, total mesorectal excision (ta-TME) in men with difficult pelvic anatomy in an attempt to optimize anal sphincteric preservation, determine the completeness of TME, and determine postoperative morbidity and mortality.

Methods

Twenty male patients囚laparoscopic-assisted transanal total mesorectal excision, A group \with difficult pelvic anatomy (narrow pelvis) who were diagnosed as rectal cancer underwent a Ta-TME surgery from January 2017 to January 2018 at Peking University Cancer Hospital. We matched these 20 patients with 2 other groups of patients who underwent either a laparoscopic transabdominal TME 囚LA group \or an open transabdominal TME $\triangle \mathrm{OP}$ group $\ a$ according to age, sex, $\mathrm{BMI}$, distance of tumor from the anal verge, and diameter of the tumor. All 3 groups of patients had undergone preoperative neoadjuvant chemoradiation therapy. The efficacy and safety of Ta-TME were evaluated according to operative time, blood loss, postoperative hospital stay, and postoperative complications. Outcomes of Ta-TME were evaluated by comparing the rate of a positive circumferential resection margin, the integrity of the TME, and the rate of sphincter preservation among the 3 groups. We also analyzed whether operative time could be shortened using a laparoscopically assisted Ta-TME through optimizing the surgical procedure.

Results

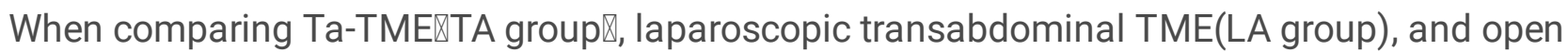
transabdominal TMEXOP group, the respective mean blood loss $(100 \mathrm{~mL}, 100 \mathrm{~mL}, 100 \mathrm{~mL}$, $p=0.335)$,postoperative hospital stay(9 days, 9 days,7 days),number of harvested lymph nodes $(7,6,7$,$) ,$ positive circumferential resection margin rate $(0 \%, 0 \%, 5 \%)$, rate of pathologic complete response $(5 \%, 10 \%, 10 \%)$, and integrity of TME showed no statistical differences across groups ( $p>0.5$ for all). In contrast, there were significant differences in operation time(302 min, $253 \mathrm{~min}, 135 \mathrm{~min}$ ),rate preservation of the anal sphincter $(100 \%, 30 \%, 45 \%)$, and the creation of a protective diverting ileostomy $(100 \%, 30 \%, 45 \%$, $p<0.05$ for all).

Conclusion

The rate of anal sphincter preservation in the Ta-TME group was considerably greater than the other groups, but the safety of the operation did not differ among the 3 groups. The Ta-TME required a diverting ileostomy in all cases, and the total operation time for Ta-TME was greater than that of laparoscopic and open transabdominal TME. We believe that experience and appropriate procedure of the steps of the Ta-TME may lead to a decrease in the operation time.

\section{Background}


It is estimated there will be about 43,000 new cases of rectal cancer in the US in 2018 , of which as many as 26,000 are likely to occur in males [1].Operative resection following the principle of a total mesorectal excision(TME),is the mainstay of treatment for patients with rectal cancer to minimize local recurrence. With $\mathrm{TME}$, the rates of local recurrence(LR) have greatly decreased from nearly $33 \%$ to less than $10 \%$ [2]. Recently, laparoscopic surgery has been used widely in clinical practice and often provides better visualization and potentially better functional outcomes than open surgery. In the traditional "up-to-down "of the laparoscopic and open transabdominal approach when performing the TME, surgeons may find it difficult to perform the TME in certain clinical scenarios, including male patients with a typical narrow pelvis, patients with a high body mass index(BMI),or when there is edema and/or fibrosis after preoperative chemoradiation, especially with bulky middle to distal rectal tumors [3]; in these situations the exposure and dissection of the distal margin of either the rectal wall or the mesorectum might be compromised without clear exposure. Additionally, conversion from laparoscopic to open surgery occurs in $10-30 \%$ of patients, often leading to longer operation times and adverse intraoperative events [4-6].Therefore, a new technical approach that facilitates exposure of the distal part of the resection/dissection is greatly needed.

Combined Trans Abdominal-Trans Anal surgery(TATA) was introduced by Marks in the 1990s to preserve the sphincter through the distal to proximal approach [7].This concept, even with the open surgery approach, laid a good foundation for later improvements, but not many surgeons have adopted this approach as a technique for patients with a narrow pelvis and poor visualization. The feasibility of dissection from the distal side proximally has been demonstrated. We wondered if a transanal approach might eliminate the difficulties of visualization and dissection of patients with a narrow or otherwise difficult rectal pelvic anatomy encountered during open and laparoscopic rectal surgery. Fortunately, natural orifice transluminal endoscopic surgery(NOTES), integrating TATA and transanal endoscopic microsurgery(TEM) demonstrated that TME can be completed via a transanal approach and had the advantage of no scar. The first case of transanal total mesorectal excision(Ta-TME) was performed by Sylla et al. In 2010 [12\}; then, the multiluminal, single port initiated by Atallah et al established a more technically appealing approach to TaTME [13].Available data have demonstrated the non-inferiority of Ta-TME compared to laparoscopic surgery in terms of the distal margin, the circumferential resection margin(CRM), and the integrity of the specimen [14].Regarding long-term outcomes, most importantly, the local recurrence rate was $7.4 \%$ among 373 patients with a median follow-up of 5.5 years, distant metastasis occurred in $19.5 \%$, and the 5 -year overall survival was $90 \%[15]$.

Most studies enrolled patients with either middle or low rectal cancer. Thus, there was little evidence to compare the outcomes of locally advanced, bulky distal rectal cancer in patients with a narrow pelvis. Most locally advanced rectal cancers are also treated with neoadjuvant chemoradiation, which makes the surgery even more difficult. Therefore, we evaluated the short-term outcomes of Ta-TME in this subgroup of male patients with a narrow pelvis who had undergone neoadjuvant chemoradiation therapy compared to patients undergoing the more classic laparoscopic or open transabdominal dissection. All operations in the Ta-TME group were performed by 2 experienced senior surgeons (Wu AW, Wang L)trained in the UK and the Netherlands.

\section{Methods}


A prospective data base was established for this Ta-TME program at Peking University Cancer Hospital from June 2017 to January 2018. Only patients matching all the following criteria were included for analysis: males with biopsy-proven, distal rectal adenocarcinoma( less than $5 \mathrm{~cm}$ from the distal margin of the mass to the anal verge), locally advanced rectal cancer having undergone neoadjuvant chemoradiation, tumor diameter of less than $4 \mathrm{~cm}$, a narrow pelvis (interischial tuberosity distance of less than $10 \mathrm{~cm}$ ), and a BMI greater than $26 \mathrm{~kg} / \mathrm{m} 2$. This study was approved by the ethics committee of Peking University cancer hospital (approval no. 2017YJZ42-GZ01).

Informed written consent was obtained from all patients. Patients with a prior history of other malignancies, recurrent or metastatic lesions, KPS $\leq 60$, poor anal function, concomitant inflammatory bowel disease, or familial adenomatous polyposis or multiple colorectal polyps were excluded from analysis. Cases of open and laparoscopic surgery were matched from the database by age, sex, BMI, distance between the interischial tuberosity, and neoadjuvant chemoradiation.

\section{Technique of Ta-TME}

All patients in the three groups received bowel preparation the day before the operation, and intravenous antibiotics were prophylactically administered perioperatively.

Laparoscopic-assisted Ta-TME: Patients were placed in the lithotomy position; the skin was prepared and draped to allow access to both the perineum and abdomen. First, the rectal dissociation was performed under laparoscopy in accordance with the TME principle囚Low ligation of the inferior mesenteric artery and preservation of the left colonic artery were usually performed, but complete mobilization of the splenic flexure was not routine. No rectal dissection was done before the transanal dissection. After digital dilation of the anal sphincter, the port was inserted transanally, and sometimes dissection of the install rectum and purse-stringing were done under direct vision. The instruments included either a transanal endoscopic operation (TEO) device (Karl Storz GmbH\&Co.KG, Tuttlingen , Germany) or Star port (Shin aide Co., Xiamen, China) after exposure of the anal canal. The distal rectal dissection, including the TME, was begun posteriorly in most cases, followed by a symmetrical bilateral dissection, and finally the anterior dissection. Once the peritoneal reflection was opened, the abdominal group then assisted in freeing to allow removal of the specimen through the anus, occasionally from a small incision when the tumor was too large. The colorectostomy anastomosis was performed either by hand sewing or with a stapler VIA. A protective loop ileostomy was constructed in all cases of T-TME.

Transabdominal TME: Laparoscopic and open surgery were performed routinely following the principles of TME resection principle. Surgeons decided whether to use a protective stoma; these stomas were generally closed 3-6 months after the operation. Postoperative complications were graded according to the DhindoClavien classification[16].

All patients were followed up at 1 month postoperatively and then every 3 months for 2 years. Digital examination was done routinely to assess the status of the anastomosis. Adjuvant chemotherapy was adopted according to the NCCN guideline.

\section{Statistics}


The $8^{\text {th }}$ AJCC/UICC TNM staging system was used [17]. A positive CRM was defined as tumor lessthan $1 \mathrm{~mm}$ from the margin, and Nagtegaal's 3-scale grading was used to evaluate the quality of the dissected specimen $[18,19]$. All analyses were performed using SPSS version 20.0( SPSS, Inc., Chicago, IL). Independent, nonparametric Kruskal-Wallis tests were used to compare the groups, and t-tests were used for continuous parameters which were represented either as mean values +/- standard deviations or as median values with the range; a p-value of less than 0.05 was used as the cutoff for statistical significance.

\section{Results}

From June 2016 to January 2018, 20 patients met the criteria mentioned above who underwent Ta-TME. Another 2 groups of 20 patients each who had undergone a transabdominal TME via open and a laparoscopic approach during the same time interval with matched parameters were used as controls. All patients were male and received neoadjuvant chemoradiation. The median ages were $57 \pm 11,59 \pm 10$, and $61 \pm 9$ in the Ta-TME, laparoscopic, and open groups, respectively. Median BMls were $27.7 \mathrm{~kg} / \mathrm{m}^{2}, 27.8 \mathrm{~kg} / \mathrm{m}^{2}$, and $28.4 \mathrm{~kg} / \mathrm{m}^{2}$, respectively. Median distances from the anal verge to the distal border of the tumor were 4 $\mathrm{cm}, 4 \mathrm{~cm}$, and $4 \mathrm{~cm}$, respectively. Median distances between the 2 is chialtuberosities were $9.3 \pm 0.53 \mathrm{~cm}, 9.1$ $\pm 0.6 \mathrm{~cm}$, and $9.3 \pm 0.2 \mathrm{~cm}$, respectively.

Table 1 presents the baseline information for the TA group. The mean blood loss was $100 \mathrm{~mL}$ (range 50 to $200 \mathrm{~mL}$ ), and operation time was $302 \mathrm{~min}$ (range 215-405 min). 95هष19/20区 were Stage III patients. A median of seven lymph nodes were harvested. At a median follow-up of 8.5 months(range 3 to 18), all patients in the Ta-TME group were alive and without disease, 1 was lost at follow-up, 18 had no evidence of disease, 1 had lymph node metastasis to the right supraclavicular area but is still alive, and 2 had undergone stoma closure; there were no local recurrences noted at the end of follow up.

Table 2 shows the comparisons of the 3 groups. There were no significant differences among the 3 groups in terms of blood loss, postoperative hospital stay, number of lymph nodes harvested, positive lymph nodes, CRM positivity, rates of a complete pathologic response to the neoadjuvant therapy, and the quality of TME specimen. There were, however, statistically significant differences found in operation time, the rate of anal sphincter-preservation, and the use of a protective, proximal diverting stoma (ileostomy). The Ta-TME group had a much superior rate of intraoperative preservation of the anal sphincter ( $100 \%$ vs. $30 \%$ vs. $45 \%$; $p<0.05)$, but also had longer operation times( $302 \mathrm{~min}$ vs. $253 \mathrm{~min}$ vs. 135min; $p<0.05$ ), and a need for a protective proximal diverting stoma (100\%vs. $30 \%$ vs.45\%; p<0.05). Postoperative complications occurred in 5 of the 20 cases in the Ta-TME group. Three had pelvic infections and were discharged on postoperative day after receiving intravenous antibiotics for 5 days (Grade II); one other patient had postoperative ileus and recovered after conservative treatment being discharged on postoperative day. The laparoscopic group had 4 complications ( 3 grade II complications involving and 1 grade III anastomotic leakage necessitating a second laparotomy), while the OP group had 5 complications involving 5 infections all treated successfully with antibiotics,(grade II )complications, respectively.

\section{Discussion}


This study found that laparoscopic-assisted Ta-TME had great advantages over a totally laparoscopic and open transabdominal TME insphincter-preservation, especially for patients with difficult operative conditions, such as bulky distal rectal carcinomas in male patients with a narrow pelvis or a high BMI who were treated with neoadjuvant chemoradiation therapy.

In the Ta-TME group, intraoperative sphincter preservation was achieved in $100 \%$ of the 20 patients, in contrast to rates of sphincter preservation of only $30 \%$, and $45 \%$ of patients in the transabdominal TME laparoscopy and open surgery groups. Because one patient in the Ta-TME group required a permanent colostomy after developing an anastomotic leak, the ultimate rate of sphincter preservation was $95 \%$ to date. Nevertheless, the long-term rate of sphincter preservation was much greater with the Ta-TME approach compared to the other groups. Transabdominal resection of the distal rectum, either open or laparoscopic, usually uses the double-stapler technique, which necessitates the ability to transection the distal rectum with the stapler. Unfortunately, this maneuver is difficult or even impossible with current surgical instruments when the pelvis is deep and narrow. Besides, the anastomosis may be impossible under direct visualization. Most importantly, for patients with difficult pelvis conditions the so-called distal margin is generally over estimated, and squeezing the tumor tissue during exposure is inevitable. Does not conform to the principle of no tumor. Rouanet et al. Reported on 30 men with advanced or recurrent low rectal tumors associated with unfavorable anatomic or tumor characteristics who underwent a sphincter-sparing, transanal endoscopic proctectomy[3]. Though the operated group included recurrent rectal cancer, 78\% still had sphincter preservation at a median follow-up of 21 months. Local anatomy(deep narrow pelvis, fatty mesorectum), male sex, high BMl, and certain features of the tumor(anterior location and large tumors) are independent risk factors for conversion, operation time, morbidity, and noncurative resection. By overcoming existing restrictions, Ta-TME may make sphincter-preservation surgery both easier and more successful. Factors such as a narrow male pelvic and a high BMI may lead to an inevitable permanent colostomy after the conversion from laparoscopic to open surgery.

Though a Ta-TME is technically feasible, the quality of surgical specimens-especially whether a complete excision of the mesorectum can be obtained and whether it might pose additional risks of local recurrencehas been questioned[19,20]. A positive CRM and its integrity are important factors in local recurrence [21]. Available data have shown that negative CRMs were present in $87.9-97.0 \%$ of open resections and $90.5-$ $97.1 \%$ of laparoscopic resections $[22,23]$. Buchs etal. reported that the traditional surgical approaches may lead to a greater rate of positive CRMs for tumors less than $3 \mathrm{~cm}$ from the dentate line [24]. Ina study of 186 cases, Lacy found positive CRMs present in $8.1 \%$ of patients [25]. Theoretically, a more meticulous dissection as allowed because of better visualization by the Ta-TME approach may be very helpful for achieving a negative CRM for these distal rectal tumors; indeed, this was verified by Denost etal. in a randomized clinical trial [26]. That trial enrolled 100 patients between 2008 and 2012 with distal rectal cancers ( $<6 \mathrm{~cm}$ from the anal verge) otherwise suitable for sphincter preservation. The primary endpoint was the quality of the resection(rate of a positive CRM, the grade of the integrity of the TME, and the ability to remove the local lymph nodes). The rate of a positive CRM decreased markedly after a transperineally dissection compared to a transabdominal distal rectal dissection ( $4 \%$ versus $18 \% ; p=0.025$ ). In our cohort, all patients in the Ta-TME group achieved a negative CRM and complete specimens. Our ability to accomplish this success rate was unexpected, because it was the first cohort of patients in whom we had performed this 
operation, and it might be attributable to either our prior extensive experience with dissection of distal rectal tumors or to the small sample size.

Safety is important for patients and surgeons when a new technique is introduced, especially during the period of the learning curve. This technique has drawn much attention, and various training courses have been introduced in Europe and the United States. In the study by Atallah and colleagues, the mean blood loss for 20 patients was $153 \mathrm{~mL}$ [27]. In a registry study of 720 cases, $61.2 \%$ of patients had blood loss of less than $100 \mathrm{~mL}$ and only $1 \%$ of these patients had a blood loss of greater than $1 \mathrm{~L}[28]$. Complications, such as ureteral injury or massive hemorrhage, are among the unique complications that could been countered during this kind of surgery. The need for a non-planned re-operation, a grade III complication, indicates a serious complication. Burke etal. Reported are operation rate of $12 \%$ among their first 50 patients, mainly due to ileostomy dysfunction, anastomotic leak, or a pelvic collection [29].In a study of 720 cases[28], however, postoperative mortality was generally quite low (about $0.5 \%$ ). In our cohort, no deaths occurred. Current available data indicate that Ta-TME is a safe operative technique. Indeed, in our study, the median postoperative hospital stay was 9 days and comparable to those in the laparoscopic or open surgery groups. This result is consistent with the results reported by Araujo etal. for 150 cases[30].

A sufficient and appropriate lymph adenectomy is necessary for the accurate staging of rectal cancer and also indicates the quality of the resection. Inadequate dissection of the mesorectum leads to a greater risk of residual disease and then an increased and unsatisfactory rate of local recurrence [31-34]. After neoadjuvant chemoradiation, patients have fewer lymphnodes [35], but in our study, the median number of lymphnodes harvested was not different between the 3 groups $(7,6$, and 7 nodes per resection.).

Regarding operation time, the Ta-TME group had a significantly longer median operation time compared to the open and laparoscopic groups(302 min, $253 \mathrm{~min}$, and $135 \mathrm{~min} ; p<0.001$ ). The shortest times for each of these groups were $215 \mathrm{~min}, 105 \mathrm{~min}$, and $88 \mathrm{~min}$, respectively. This finding could be related to the learning curve. There were $2 \mathrm{~g}$ roups in the Ta-TME operations-the abdominal and perineal. The operation can be completed by 1 or 2 teams of surgeons, concurrently or sequentially. During the learning curve period, the choice of operational platform is of utmost importance. Moreover, the establishment of the pneumo-rectum with a conventional device or with TEM(transanal endoscopic microsurgery) platform or Tamis(transanal minimally invasive surgery) platform differed greatly in shortening operation times. The longest Ta-TME operation lasted $405 \mathrm{~min}$, comparable to that reported by Araujo, Simillis AND COLLEGUES $[29,36]$. The number of cases needed to complete the learning period has been estimated as $20-40$, depending on the surgeon's prior experience and the operating room supportive team [24]. Optimization of protocols is necessary.

Our study had several limitations. First, although the sample size was small, all patients had a difficult anatomy. Second, all cases of the Ta-TME were conducted within our learning-curve period. The longer operation times, complication patterns, and rates, as well as the longer hospital stays might produce some bias. Third, this was a case-matched study that only incorporated certain factors that theoretically affected outcomes for rectal cancer patients. Some other parameters that might have made the 2 control groups either better matched or were indicative of poor matches might exist that were not included in the analysis. Finally, follow-up times were short and could not provide better long-term oncologic outcomes. Therefore, 
long-term follow-up is needed in terms of local recurrence, actual sphincter-preservation after stoma closure, and patient-reported rectal function.

In conclusion, compared to a transabdominal open and laparoscopic approach, Ta-TME appears to be superior insphincter preservation for patients with distal rectal cancer, especially when patients are male, have a narrow pelvis , have a high $\mathrm{BMI}$, and have had a course of neoadjuvant chemoradiation therapy. Our study strongly suggests that Ta-TME is a safe procedure in experienced hands that may benefit from structured training for shorter operation times. It is clear, however, that the value of Ta-TME still needs to be evaluated through larger randomized trials[2].

\section{Abbreviations}

TME: total mesorectal excision

ta-TME: transanal total mesorectal excision

LA group: laparoscopic transabdominal TME

OP group: open transabdominal TME

$L R$ : local recurrence

BMI: body mass index

TATA: Trans Abdominal-Trans Anal surgery

NOTES: natural orifice trans/uminal endoscopic surgery

TEM: transanal endoscopic microsurgery

CRM: circumferential resection margin

KPS: Karnofsky score

TEO: transanal endoscopic operation

\section{Declarations}

\section{Ethics approval and consent to participate}

This study was approved by the ethics committee of Peking University cancer hospital (approval no. 2017YJZ42-GZ201).

\section{Consent for publication}

All authors have agreed to the publication of this manuscript. 


\section{Competing interests}

The authors have declared that no competing interests exist.

\section{Availability of data and materials}

The datasets used in the current study are available from the corresponding author on reasonable request.

\section{Funding}

This work was supported the National Natural Science Foundation of China (81773214).

Fund funder: Aiwen Wu: conception and design of the study, draft and final approval of the manuscript. Correspondence and requests for materials should be addressed to Aiwen Wu (drwuaw@sina.com, Fax: 8610-88122437; Tel: 8610-88196086.)

\section{Author Contributions}

A.W.W: conception and design of the study, draft and final approval of the manuscript; Y.J.L and G.L.H: collection of the data, draft the manuscript; L.W, Q.S.D, X.Z.L, J.H.L, X.Z, Y. Z, and Y.F.Yषquality control of the study especially the surgery part, acquisition of data; T.T.S: study design and statistical analysis. All authors approved the final manuscript.

\section{Conflict of interest declaration:None.}

\section{Acknowledgments:}

We thank Center for the department of Clinical Epidemiology, Peking University Cancer Hospital \& Institute for the assistance in data collection and analysis. Part of the analysis was performed on the Computing Platform of School of Public Health, Peking University.

\section{References}

1. Siegel, R.L., K.D. Miller, and A. Jemal, Cancer statistics, 2018. CA Cancer J Clin, 2018. 68(1): p. 7-30.

2. van Gijn, W., et al., Preoperative radiotherapy combined with total mesorectal excision for resectable rectal cancer: 12-year follow-up of the multicentre, randomised controlled TME trial. The Lancet Oncology, 2011. 12(6): p. 575-582.

3. Rouanet, P., et al., Transanal endoscopic proctectomy: an innovative procedure for difficult resection of rectal tumors in men with narrow pelvis. Dis Colon Rectum, 2013. 56(4): p. 408-15.

4. Mh, V.D.P., et al., Laparoscopic versus open surgery for rectal cancer (COLOR II): short-term outcomes of a randomised, phase 3 trial. Lancet Oncology, 2013. 14(3): p. 210-8.

5. Penninckx, F., et al., Outcome following laparoscopic and open total mesorectal excision for rectal cancer. British Journal of Surgery, 2013. 100(10): p. 1368-1375. 
6. Jayne, D.G., et al., Five-year follow-up of the Medical Research Council CLASICC trial of laparoscopically assisted versus open surgery for colorectal cancer. British Journal of Surgery, 2010. 97(11): p. 16381645.

7. Marks, G., M. Mohiuddin, and J. Rakinic, New hope and promise for sphincter preservation in the management of cancer of the rectum. Semin Oncol, 1991. 18(4): p. 388-98.

8. Buess, G., et al., Endoscopic surgery in the rectum. Endoscopy, 2008. 17(01): p. 31-35.

9. Wilk, P.J., Method for use in intra-abdominal surgery. 1995, US.

10. Kalloo, A.N., et al., Flexible transgastric peritoneoscopy: a novel approach to diagnostic and therapeutic interventions in the peritoneal cavity. Gastrointestinal Endoscopy, 2004. 60(1): p. 114-117.

11. Marescaux, J., et al., Surgery Without Scars: Report of Transluminal Cholecystectomy in a Human Being. Archives of Surgery, 2007. 142(9): p. 823-826.

12. Sylla, P., et al., NOTES transanal rectal cancer resection using transanal endoscopic microsurgery and laparoscopic assistance. Surgical Endoscopy, 2010. 43(3): p. 1205-1210.

13. Atallah, S., M. Albert, and S. Larach, Transanal minimally invasive surgery: a giant leap forward. Surg Endosc, 2010. 24(9): p. 2200-5.

14. Velthuis, S., et al., Transanal versus traditional laparoscopic total mesorectal excision for rectal carcinoma. Surg Endosc, 2014. 28(12): p. 3494-9.

15. Marks, J.H., et al., Long-term outcomes by a transanal approach to total mesorectal excision for rectal cancer. Surg Endosc, 2017. 31(12): p. 5248-5257.

16. Clavien PA, Barkun J, de Oliveira ML, et al.: The Clavien-Dindo classification of surgical complications: five-year experience. Annals of surgery 2009;250:187-196.

17. Weiser, M.R., AJCC 8th Edition: Colorectal Cancer. Ann Surg Oncol, 2018.

18. Quirke, P., et al., LOCAL RECURRENCE OF RECTAL ADENOCARCINOMA DUE TO INADEQUATE SURGICAL RESECTION : Histopathological Study of Lateral Tumour Spread and Surgical Excision. Lancet 1986. 2(8514): p. 996.

19. Nagtegaal, I.D., et al., Macroscopic evaluation of rectal cancer resection specimen: clinical significance of the pathologist in quality control. Journal of Clinical Oncology Official Journal of the American Society of Clinical Oncology, 2002. 20(7): p. 1729.

20. Quirke, P., et al., Effect of the plane of surgery achieved on local recurrence in patients with operable rectal cancer: a prospective study using data from the MRC CR07 and NCIC-CTG CO16 randomised clinical trial. Lancet, 2009. 373(9666): p. 821-8.

21. Nagtegaal, I.D. and P. Quirke, What is the role for the circumferential margin in the modern treatment of rectal cancer? J Clin Oncol, 2008. 26(2): p. 303-12.

22. Stevenson, A.R., et al., Effect of Laparoscopic-Assisted Resection vs Open Resection on Pathological Outcomes in Rectal Cancer: The ALaCaRT Randomized Clinical Trial. JAMA, 2015. 314(13): p. $1356-63$.

23. Fleshman, J., et al., Effect of Laparoscopic-Assisted Resection vs Open Resection of Stage II or III Rectal Cancer on Pathologic Outcomes: The ACOSOG Z6051 Randomized Clinical Trial. JAMA, 2015. 314(13): p. 1346-55. 
24. Buchs, N.C., et al., A two-centre experience of transanal total mesorectal excision. Colorectal Dis, 2016. 18(12): p. 1154-1161.

25. de Lacy, F.B., et al., Transanal total mesorectal excision: pathological results of 186 patients with mid and low rectal cancer. Surg Endosc, 2017.

26. Denost, Q., et al., Perineal transanal approach: a new standard for laparoscopic sphincter-saving resection in low rectal cancer, a randomized trial. Ann Surg, 2014. 260(6): p. 993-9.

27. Atallah, S., et al., Transanal minimally invasive surgery for total mesorectal excision (TAMIS-TME): results and experience with the first 20 patients undergoing curative-intent rectal cancer surgery at a single institution. Tech Coloproctol, 2014. 18(5): p. 473-80.

28. Penna, M., et al., Transanal Total Mesorectal Excision: International Registry Results of the First 720 Cases. Ann Surg, 2017. 266(1): p. 111-117.

29. Burke, J.P., et al., Transanal total mesorectal excision for rectal cancer: early outcomes in 50 consecutive patients. Colorectal Dis, 2016. 18(6): p. 570-7.

30. Araujo, S.E., et al., Transanal total mesorectal excision: a systematic review of the experimental and clinical evidence. Tech Coloproctol, 2015. 19(2): p. 69-82.

31. Cianchi, F., et al., Lymph node recovery from colorectal tumor specimens: recommendation for a minimum number of lymph nodes to be examined. World Journal of Surgery, 2002. 26(3): p. 384.

32. Pocard, M., et al., Assessing the effectiveness of mesorectal excision in rectal cancer. Diseases of the Colon \& Rectum, 1998. 41(7): p. 839-45.

33. Goldstein, N.S., Lymph node recoveries from 2427 pT3 colorectal resection specimens spanning 45 years: recommendations for a minimum number of recovered lymph nodes based on predictive probabilities. American Journal of Surgical Pathology, 2002. 26(2): p. 179.

34. JE, T., et al., Impact of number of nodes retrieved on outcome in patients with rectal cancer. Journal of Clinical Oncology, 2001. 19(1): p. 157.

35. Morcos, B., et al., Lymph node yield in rectal cancer surgery: effect of preoperative chemoradiotherapy. Eur J Surg Oncol, 2010. 36(4): p. 345-9.

36. Simillis, C., et al., A systematic review of transanal total mesorectal excision: is this the future of rectal cancer surgery? Colorectal Dis, 2016. 18(1): p. 19-36.

\section{Tables}

Table 1. Basic information for patients in the Ta-TME group. 


\begin{tabular}{|c|c|c|c|c|c|c|c|c|c|}
\hline No. & $\begin{array}{l}\text { Age } \\
\text { (y) }\end{array}$ & BMI & $\begin{array}{l}\text { DAV } \\
(\mathrm{cm})\end{array}$ & $\begin{array}{l}\text { Clinical } \\
\text { stage }\end{array}$ & $\begin{array}{l}\text { IITD } \\
(\mathrm{mm})\end{array}$ & $\begin{array}{l}\text { Operation } \\
\text { time } \\
\text { (min) }\end{array}$ & $\begin{array}{l}\text { Blood } \\
\text { loss } \\
(\mathrm{mL})\end{array}$ & $\begin{array}{l}\text { Hospital } \\
\text { stay (d) }\end{array}$ & $\begin{array}{l}\text { Postoperative } \\
\text { complication }\end{array}$ \\
\hline 1 & $\begin{array}{l}40- \\
59\end{array}$ & 31 & 5 & T3N2bM0 & 99 & 274 & 100 & 7 & none \\
\hline 2 & $\begin{array}{l}70- \\
79\end{array}$ & 36. & 3 & $\mathrm{~T} 4 \mathrm{~N}+\mathrm{M} 0$ & 94 & 405 & 100 & 10 & none \\
\hline 3 & $\begin{array}{l}50- \\
59\end{array}$ & 27 & 2 & $\mathrm{~T} 3 \mathrm{~N}+\mathrm{M} 0$ & 95 & 342 & 200 & 7 & none \\
\hline 4 & $\begin{array}{l}60- \\
69\end{array}$ & 27 & 5 & $\mathrm{~T} 3 \mathrm{~N}+\mathrm{M} 0$ & 91 & 290 & 100 & 9 & none \\
\hline 5 & $\begin{array}{l}30- \\
39\end{array}$ & 26. & 3 & $\mathrm{~T} 3 \mathrm{~N}+\mathrm{M} 0$ & 91 & 283 & 100 & 12 & none \\
\hline 6 & $\begin{array}{l}60- \\
69\end{array}$ & 27 & 5 & T2N2M0 & 98 & 245 & 100 & 9 & none \\
\hline 7 & $\begin{array}{l}50- \\
59\end{array}$ & 28 & 2 & T3N2bM0 & 100 & 364 & 200 & 7 & $\begin{array}{l}\text { pelvic } \\
\text { infection }\end{array}$ \\
\hline 8 & $\begin{array}{l}50- \\
59\end{array}$ & 27 & 4 & $\mathrm{~T} 3 \mathrm{~N}+\mathrm{MO}$ & 98 & 400 & 100 & 9 & none \\
\hline 9 & $\begin{array}{l}60- \\
69\end{array}$ & 30 & 5 & T3N2bM0 & 95 & 364 & 100 & 9 & $\begin{array}{l}\text { pelvic } \\
\text { infection }\end{array}$ \\
\hline 10 & $\begin{array}{l}60- \\
69\end{array}$ & 27 & 4 & T3N1M0 & 88 & 270 & 100 & 9 & none \\
\hline 11 & $\begin{array}{l}60- \\
69\end{array}$ & 26 & 4 & $\mathrm{~T} 3 \mathrm{~N}+\mathrm{M} 0$ & 91 & 262 & 50 & 12 & none \\
\hline 12 & $\begin{array}{l}50- \\
59\end{array}$ & 28 & 5 & T3N2M0 & 86 & 392 & 100 & 14 & none \\
\hline 13 & $\begin{array}{l}30- \\
39\end{array}$ & 29 & 3 & T3aN2bM0 & 91 & 266 & 100 & 5 & none \\
\hline 14 & $\begin{array}{l}40- \\
49\end{array}$ & 28 & 5 & T3N1M0 & 97 & 317 & 100 & 13 & $\begin{array}{l}\text { pelvic } \\
\text { infection }\end{array}$ \\
\hline 15 & $\begin{array}{l}40- \\
49\end{array}$ & 28 & 4 & $\mathrm{~T} 3 \mathrm{~N}+\mathrm{MO}$ & 93 & 375 & 100 & 7 & ileus \\
\hline 16 & $\begin{array}{l}60- \\
69\end{array}$ & 27 & 4 & T3N2bM0 & 99 & 293 & 100 & 9 & none \\
\hline 17 & $\begin{array}{l}60- \\
69\end{array}$ & 29 & 5 & $\mathrm{~T} 4 \mathrm{aN}+\mathrm{M} 0$ & 92 & 337 & 100 & 7 & none \\
\hline 18 & $\begin{array}{l}60- \\
69\end{array}$ & 27 & 4 & T3N1M0 & 91 & 271 & 100 & 7 & none \\
\hline 19 & $\begin{array}{l}70- \\
79\end{array}$ & 29 & 5 & T3N1M0 & 78 & 310 & 100 & 15 & $\begin{array}{l}\text { anastomosis } \\
\text { leak }\end{array}$ \\
\hline
\end{tabular}




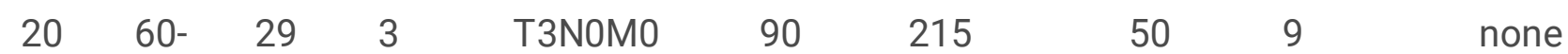
69

Note: DAV: distance from the anal verge to the inferior border of the tumor; IITD: interischial tuberosity distance. 